\title{
ArcheoSciences
}

Revue d'archéométrie

$31 \mid 2007$

Varia

\section{L'apport de l'analyse morphologique, microscopique et chimique des scories en forme de culot à la restitution des activités de forge}

The contribution of the morphological, microscopical and chemical analyses of hearth bottom slags to the restitution of smithing activities

Cécile Le Carlier, Marc Leroy et Paul Merluzzo

\section{OpenEdition}

Journals

Édition électronique

URL : https://journals.openedition.org/archeosciences/581

DOI : 10.4000/archeosciences.581

ISBN : 978-2-7535-1596-3

ISSN : 2104-3728

Éditeur

Presses universitaires de Rennes

Édition imprimée

Date de publication : 31 décembre 2007

Pagination : 23-35

ISBN : 978-2-7535-0618-3

ISSN : 1960-1360

Référence électronique

Cécile Le Carlier, Marc Leroy et Paul Merluzzo, « L'apport de l'analyse morphologique, microscopique et chimique des scories en forme de culot à la restitution des activités de forge ", ArcheoSciences [En ligne], 31 | 2007, document 2, mis en ligne le 31 décembre 2009, consulté le 01 février 2022. URL http://journals.openedition.org/archeosciences/581; DOI : https://doi.org/10.4000/archeosciences. 581 


\title{
L'apport de l'analyse morphologique, microscopique et chimique des scories en forme de culot à la restitution des activités de forge
}

\author{
The contribution of the morphological, microscopical and chemical analyses \\ of hearth bottom slags to the restitution of smithing activities
}

\author{
Cécile Le Carlier*, Marc LeroY* et Paul Merluzzo*
}

\begin{abstract}
Résumé : Si les différents travaux réalisés dans une forge sont assez bien connus, il est difficile de les retrouver au travers de l'analyse des seuls déchets. Ce sont pourtant ceux-ci que l'on retrouve presque exclusivement sur les ateliers dégagés au cours de fouilles archéologiques. Pour en extraire un maximum de renseignements, il est nécessaire de mettre en place une méthodologie rigoureuse d'étude, depuis l'échantillonnage sur le terrain jusqu'aux analyses chimiques en laboratoire. Les notions de quantification et de représentativité sont extrêmement importantes pour tenter de qualifier et quantifier les travaux effectués par le forgeron, pour déterminer la nature des matériaux employés et pour tenter d'estimer une quantité minimale de fer travaillé. L'étude, bien que ciblée sur les scories en culot, montre que l'ensemble des déchets produits dans un atelier doit être pris en considération lors des études paléométallurgiques si l'on veut réellement appréhender l'atelier. L'exemple du site du Puy-de-Grâce (La Tène finale) permet de documenter les apports de cette méthodologie.
\end{abstract}

Abstract: If the different works produced in a smithing workshop are well known, it is difficult to identify them only from the analyses from the wastes. However, these wastes are generally the only remnants that can be found during excavation of archaeological ironworkshops. To obtain the maximum of informations of these wastes, it is necessary to propose a rigorous methodology, from the sampling in the archaeological site to chemical analyses in laboratory. The quantification and representativeness notions are very important to qualify and quantify the works operated by the blacksmith, to determine the nature of used materials and to estimate the minimal quantity of worked iron. This study, although targeted on the tape slags, shows that it is necessary to take into account the totality of the wastes found in the archaeological smithy during the archeaometallurgical studies for a good comprehension of this smithy functioning. The Puy-de-Grâce example (France) allows documenting the contribution of this methodology.

Mots clés : Archéométallurgie du fer, Scorie en culot, Méthodologie, Pétrologie, Forge.

Key Words: Archaeometallurgy, ironworking, Hearth bottom slag, Study methods, Petrology, Smithing.

\section{INTRODUCTION}

La chaîne opératoire de la métallurgie ancienne du fer se divise en deux grandes étapes successives : la transfor- mation du minerai en fer métallique suivant la technique de la réduction directe, et la transformation de ce fer brut de réduction en fer forgeable, puis en objets ouvragés (Mangin [dir.], 2004). Si les déchets issus de l'étape de la

* Laboratoire de Métallurgies et Cultures - UMR 5060 IRAMAT LAM, avenue Charles-de-Gaulle, 54140 Jarville-la-Malgrange. (cecile_le_carlier@ hotmail.com) (marc.leroy@lam.id-net.fr) (paul.merluzo@lam.id-net.fr) 
réduction ont fait l'objet de nombreuses études aboutissant à une meilleure connaissance des systèmes techniques de production mis en œuvre, les études archéométallurgiques des déchets liés aux activités de forge (ou post-réduction) sont encore peu nombreuses. Les travaux précurseurs (Mc Donnell, 1987 ; Serneels, 1993 ; Leroy, 1997 ; Ploquin et al., 1998) abordent les questions de reconnaissance des résidus scorifiés et de leur distinction morphologique et chimique avec les scories de réduction, et jettent les bases d'une première caractérisation des scories plano-convexes en forme de culot ou calotte. Plus récemment, une véritable caractérisation des familles de déchets est entreprise à partir de l'étude complète des vestiges d'atelier (Dunikowski et al., 1996; Serneels dans Mauvilly et al., 1998; Serneels dans Chardron-Picault [dir.], 1999; Orengo et al., 2000; Orengo 2003; Serneels dans Anderson et al., 2004, Eschenlohr et al., 2007). Ce sont des études pétrographiques et chimiques couplées aux observations morphologiques. Elles tentent de comprendre la formation des scories en culot, des battitures et des chutes métalliques et essayent de les interpréter en termes de gestes opératoires, afin de pouvoir les associer à des types de travail ou d'activités.

En s'inscrivant dans cette perspective, la présente contribution propose une présentation détaillée de la méthodologie archéométrique appliquée à l'étude des scories en culot, déchets caractéristiques des travaux de post-réduction. Elle est illustrée par l'étude de cas des culots d'un atelier de La Tène finale (le Puy-de-Grâce, commune de Perpezac-le Noir, Corrèze, inédit). Des interprétations sur la nature des activités qui y ont été pratiquées et une hypothèse sur un ordre de grandeur de la masse de fer qui y a été travaillée sont présentées.

\section{LES BASES MÉTHODOLOGIQUES FONDANT L'ÉTUDE D'UN ATELIER DE FORGE}

La transformation du fer brut et l'élaboration d'objets manufacturés s'organisent en une série de manipulations au cours desquelles le fer est, de nombreuses fois, réchauffé et martelé. Le nombre et la nature des traitements pratiqués sont fonction de la nature du métal de départ, du volume et de la complexité du produit qui est réalisé (Mangin [dir.], 2004, chapitre 3). Le fonctionnement d'un atelier de forge est donc rythmé par de nombreuses séquences de travail qui vont produire des déchets pour partie similaires et pour partie spécifiques à chacune d'elles. Les exemples ethnographiques montrent que les différentes étapes de la transformation du fer peuvent être effectuées dans des ateliers différents et parfois séparés géographiquement. Si au contraire elles ont lieu dans un même atelier, les déchets des séquences de travail successives y seront mélangés et donc difficiles à discriminer. Enfin, certains travaux d'entretien, de réparation ou de récupération produisent également des déchets. C'est pourquoi, la reconnaissance de ces différents types de déchets est très difficile sur le plan archéométallurgique.

Il est important de prendre en compte le fait que l'information archéologique conservée n'est que très partielle. La collecte de cette information lors de la fouille doit donc faire l'objet d'une méthodologie appropriée basée, autant que faire ce peut, sur une approche rigoureuse : relevé systématique des divers indices et vestiges au sol; enregistrement systématique du matériel observé et collecté au sein des contextes archéologiques. De la qualité de la collecte des informations archéologiques dépend souvent la pertinence des études archéométriques. Les études ultérieures sur ce matériel ne devront jamais être déconnectées de cette information contextuelle, ni des notions de représentativité.

Pour disposer d'une vision complète des différentes catégories de scories présentes dans un atelier sidérurgique, l'observation doit porter sur le prélèvement le plus large possible. L'étude de la répartition spatiale des vestiges apporte de nombreux renseignements, car l'organisation de l'atelier n'est souvent perceptible que par la présence de déchets des séquences de la chaîne opératoire éparpillés sur le sol ou rejetés dans des fosses et des dépotoirs.

L'analyse macroscopique des déchets métallurgiques porte sur l'ensemble du matériel récolté lors de la fouille. Celui-ci fait l'objet d'un tri sur la base de différenciations morphologiques pour aboutir à une série de catégories distinctes et significatives. Ces distinctions font intervenir la morphologie et l'aspect général, ainsi que la nature des matériaux qui constituent le déchet. Elles aboutissent à des catégories de déchets caractérisant une origine technique et/ou fonctionnelle, et dans une certaine mesure un processus de fabrication identifiable: scories en culot, scories argilo-sableuses, scories denses informes, scories en petites coulures, scories ferreuses, déchets métalliques (informes, éclats, chutes, ratés, ébauches), parois de foyers, petits déchets magnétiques (battitures plates, en billes, boursouflées...).

Une quantification (dénombrement et pesage) est réalisée par catégories de matériaux et de déchets, par unité stratigraphique et par structure. Elles peuvent être regroupées le cas échéant en fonction du système d'enregistrement utilisé sur la fouille.

Chaque catégorie identifiée de déchets fait l'objet d'une description générale et les individus remarquables d'une description morphologique de détail. Ceux-ci correspondent le plus souvent aux pièces complètes ou à défaut à celles apparaissant comme les plus significatives. Des sous-catégories 
peuvent être identifiées à partir de distinctions morphologiques, dimensionnelles ou en fonction de la nature des matériaux associés. À chaque stade, les caractéristiques globales des populations sont enregistrées (morphologie, dimensions, matériaux, particularités...).

\section{LES SCORIES EN FORME DE CULOT}

\section{Généralités}

Les scories en forme de culot sont des déchets particulièrement caractéristiques des séquences de la chaîne opératoire de post-réduction. Les études archéométallurgiques déjà publiées (supra) montrent qu'ils résultent de l'accumulation de divers matériaux plus ou moins fondus, qui se solidifient dans le fond du foyer de forge sous l'orifice de la soufflerie au cours d'un ou plusieurs chauffages. Pour simplifier, ces matériaux proviennent en grande partie de l'interaction à haute température entre la croûte d'oxyde de fer qui se forme en surface du métal et les matières argilo-sableuses que le forgeron ajoute pour en limiter la formation (voir notamment Dunikowski et al., 1996). On y trouve aussi des parcelles de fer métalliques détachés de la pièce au cours de la chauffe ou des morceaux de parois du foyer qui s'effritent sous l'impact thermique. Les cendres de charbon de bois peuvent également s'intégrer à la scorie en formation.

Tel qu'il est décrit dans les manuels classiques de forge des $\mathrm{XVIII}^{\mathrm{e}}$ et $\mathrm{XIX}^{\mathrm{e}}$ siècles, le fonctionnement d'un foyer de forge est un ensemble de cycles de montée en température, de stabilisation, puis de refroidissement. Lorsque la pièce de fer qui y est chauffée a atteint la température homogène nécessaire, elle est retirée du foyer pour être travaillée sur l'enclume; la ventilation est arrêtée le temps du martelage. Durant cet arrêt, la température décroît : débute alors dans le foyer une phase de refroidissement durant laquelle la température chute progressivement. Le foyer n'est réactivé que pour un nouveau cycle de chauffage. La scorie en culot représente donc une accumulation de matière tombée dans le fond du foyer au cours d'un cycle de travail entre la mise en route de la forge et son arrêt. Ce cycle de travail peut correspondre à une unité de temps (à la fin duquel le culot est systématiquement enlevé du foyer), ou à une unité de travail (réalisation d'une même pièce ou d'un ensemble de gestes techniques dans une suite opératoire cohérente...).

Ces scories en culot peuvent présenter des morphologies et des dimensions très variées, mais le plus généralement elles ont une forme plano-convexe. En règle générale, la scorie en calotte, par son volume, son aspect, sa structure interne et sa composition chimique, reflète une partie des activités pratiquées et les matières utilisées : il s'agit donc d'un témoin privilégié, potentiellement riche en informations très diverses. On notera que si les scories de forge sont des déchets que l'on retrouve sur d'innombrables sites archéologiques, tous les travaux de forge ne produisent pas de déchets scorifiés.

\section{La méthodologie d'étude des culots de forge}

L'étude est réalisée par paliers successifs de taux de grossissement d'observation : de l'observation globale macroscopique, on se rapproche peu à peu de celle de la matière. Au fur et à mesure de l'étude, plus le niveau d'observation augmente, plus les surfaces observées directement deviennent petites et plus les temps d'examen pour une même unité de surface deviendront grands. Cela nous amène naturellement, pour chaque palier d'observation, à réduire le nombre d'échantillons, afin de ne pas multiplier à l'infini les temps d'analyses. Pour l'approche chimique, les temps de préparation et les coûts nous contraignent aussi à cette économie. Du coup, il apparaît qu'on ne peut faire l'économie d'une très bonne analyse macroscopique initiale (en relation avec le contexte archéologique), de quoi dépendra la représentativité et donc la pertinence des résultats suivants.

\section{Les observations morphologiques}

Les scories en forme de culot moulent intégralement ou partiellement le fond du foyer de forge dans lequel elles se sont formées. Une observation attentive de ces pièces peut donc nous renvoyer une image de celui-ci et des aménagements techniques éventuels (infra). Des indices convergents reconnus sur un grand nombre d'individus (dont de très rares exemplaires ont été trouvés en place dans des foyers) permettent de restituer la position originelle du culot dans le foyer de forge. Plusieurs de ces indices sont en général associés sur une même pièce :

- une bordure d'aspect plus ou moins sub-verticale paraissant en appui contre une surface;

- un morceau de paroi de foyer en terre cuite adhérent à un bord du culot;

- une forme générale du culot symétrique par rapport à un axe médian;

- un côté plus épais;

- une excroissance localisée sur une extrémité.

Ces indices permettent de restituer l'orientation du culot par rapport à l'axe de la tuyère, et dès lors d'appréhender d'une manière plus rigoureuse l'étude des aspects morphologiques et dimensionnels.

L'examen visuel d'un corpus de scories de forge montre qu'elles paraissent constituées de matériaux de nature différente, ce qui leur donne des aspects de surface et des colorations variés. On peut définir trois types de matériaux : 
- celui qui résulte du refroidissement d'un liquide silicaté, plus ou moins riche en oxydes de fer, de couleur le plus souvent gris sombre;

- celui qui résulte du ramollissement ou de la fusion de matières de nature argilo-sableuse, caractérisées par des teintes bleutées ou claires (brunâtre, beige, verdâtre, jaunâtre) et qui montrent souvent des reflets vitreux. On y observe fréquemment des grains centimétriques de roche ou d'argile non fondus;

- celui qui se présente sous un aspect externe terreux ou rouillé correspondant à une gangue d'oxydation qui recouvre les surfaces originelles, mais qui laisse parfois apparaittre des plages de scorie grise. La présence de fer métallique est souvent révélée par la présence de fissures, ainsi que par sa propriété magnétique.

V. Serneels a récemment désigné ces matériaux de la manière suivante : SGD pour scorie grise dense, SAS pour scorie argilo-sableuse, SFR pour scorie ferreuse rouillée (Fig. 1). En fonction des associations de ces trois types de matériaux et de leur proportion relative, il a proposé une classification des culots en 8 catégories (Serneels in Anderson et al., 2004).

Pour chacune des catégories, une sélection d'échantillons représentatifs est effectuée pour des investigations plus approfondies (sections, examens microscopiques, identification minéralogique optique et chimique, analyses chimiques).

\section{Observations macroscopiques des sections sciées}

Les échantillons sont sciés, puis polis afin de pouvoir observer la surface au microscope. La structure interne de ces déchets, résultant d'une succession de gestes techniques variés (supra), est très complexe. C'est pourquoi l'observation macroscopique de la section sciée est une étape extrêmement importante car elle va permettre de repérer les zones de différentes natures constituant le culot (Fig. 2). Ce travail permet également d'observer l'agencement de ces zones entre elles.
Cette délimitation est indispensable si l'on souhaite réaliser une étude chimique. Chaque zone va apporter une contribution chimique différente. Pour être représentative, l'analyse chimique ne devra pas concerner les zones voisines. Sinon, ces analyses chimiques seront, au mieux représentatives de l'ensemble du déchet, sinon représentatives d'une partie de celui-ci, mais ne pourront pas être utilisés pour tenter de connaître la nature des matériaux d'origine, ni pour suivre une éventuelle évolution au sein du culot. Ce repérage et cette délimitation se font également grâce aux observations microscopiques (type de phases minéralogiques, concentration et orientation de celles-ci). Ces observations microscopiques vont contribuer à déterminer la nature de la zone concernée (nature de l'ajout, zone scoriacée, présence ou non de croûtes d'oxydes, etc.).

\section{Observations microscopiques des sections sciées}

Les matériaux initiaux qui vont participer à la constitution des culots sont essentiellement : des matériaux argilosableux apposés par le forgeron sur le métal, les oxydes de fer provenant de l'oxydation à chaud du métal chauffé, le fer métallique provenant de la fusion partielle du métal en cours de chauffe. Parmi ces matériaux, les deux derniers sont synonymes de hautes températures. La présence dans les culots de zones à fortes concentrations de wustite, indique un phénomène d'oxydation à chaud du métal travaillé non négligeable. Globalement, ce phénomène dépendra de certains paramètres : la température de chauffe, le temps de maintien à ces températures, la grandeur des surfaces chauffées. L'oxydation sera d'autant plus importante que les températures de chauffe seront élevées, les temps de maintien à ces températures longs et les surfaces chauffées importantes.

La pratique des ajouts, en dehors ou directement dans le foyer, est bien connue dans les manuels de forgeron (Dunikowski et al., 1996). Elle est destinée à protéger de l'oxydation les surfaces du fer au cours de la chauffe ou à éliminer
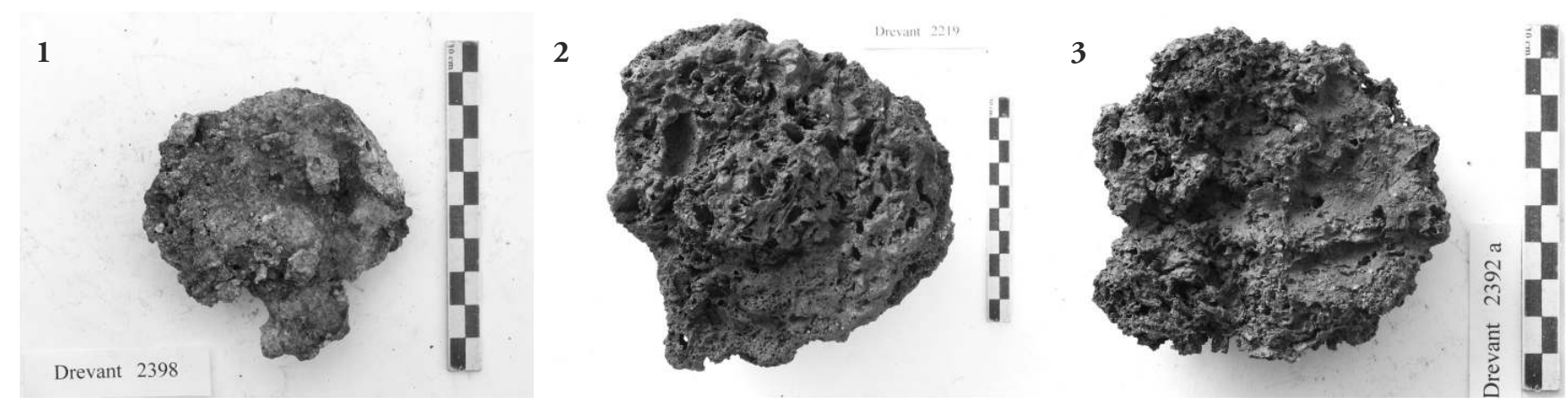

Figure 1 : Exemples d'aspect morphologique de scories argilo-sableuses SAS (1), de scories grises denses SGD + SAS (2) et de scories ferreuses rouillées SFR (3).

Figure 1: Example of morphological aspect of siliceous slags $S A S$ (1), grey compact slags $S G D+S A S$ (2) and iron rushed slags $S F R$ (3). 


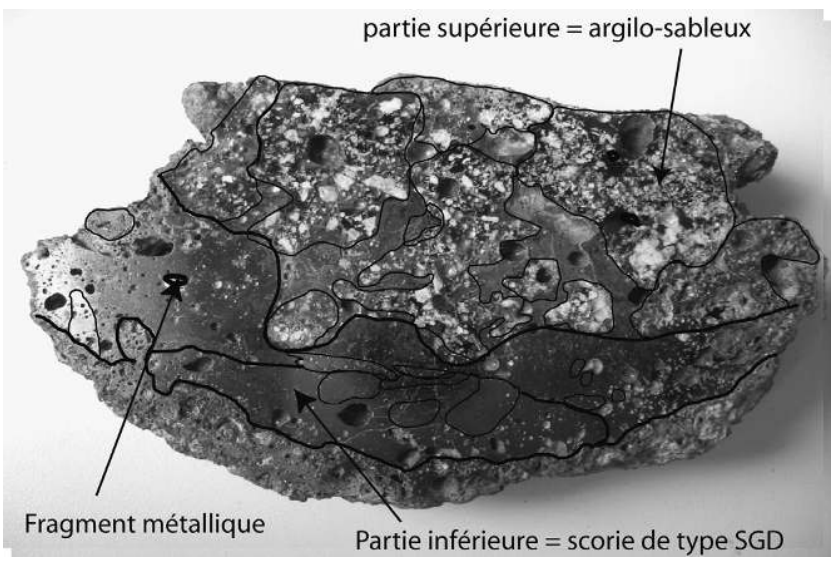

Figure 2 : Exemple d'observations réalisées sur une section sciée de culot.

Figure 2: Example of observations realized in a slag sawed section.

de ces mêmes surfaces la croûte d'oxydes qui s'y forme, avant les opérations de soudure. Ces ajouts sont de natures diverses utilisés en solution dans un liquide (généralement de l'eau) ou à sec. Les plus cités sont la barbotine de terre argileuse, la terre argileuse sèche en poudre, le sable, le grès pilé... Des mélanges plus complexes sont également évoqués. Lors du martelage cet enduit sera facilement évacué par pression pour laisser les surfaces à nu se souder. C'est l'interaction de ces matériaux avec le métal que va produire la matière scoriacée constitutive des culots : le produit silicaté qui en résulte tend à s'écouler de la pièce vers le fond du foyer. Il peut emporter avec lui des reliques de croûtes d'oxydes de fer formées sur la pièce métallique, voire même des fragments métalliques détachés de la pièce principale. Dans les culots, on peut donc observer des matériaux peu ou pas fondus (argilo-sableux, croûtes d'oxydes et métal) encore visibles : ce sont les matériaux d'origine autrement appelés " reliquats ". Ces matériaux voisinent avec des zones où tous les apports ont complètement fondus en une masse scorifiée.

\section{Les zones essentiellement composées de matière argilo- sableuse peu fondue}

Le faciès argilo-sableux correspond à ces apports en cours de fusion, mais non mélangés aux oxydes de fer. Ils peuvent être fortement fondus au point qu'il ne reste que quelques grains de quartz dans une matrice vitrifiée (Fig. 3). D'autres peuvent n'être que partiellement fondus et il est alors possible de reconnaître la nature de la roche employée.

\section{Les croûtes d'oxydes et les battitures}

Les croûtes qui se forment à la surface du métal peuvent être constituées uniquement d'oxydes de fer ou peuvent être un mélange entre les oxydes de fer et l'ajout déposé sur l'ob-

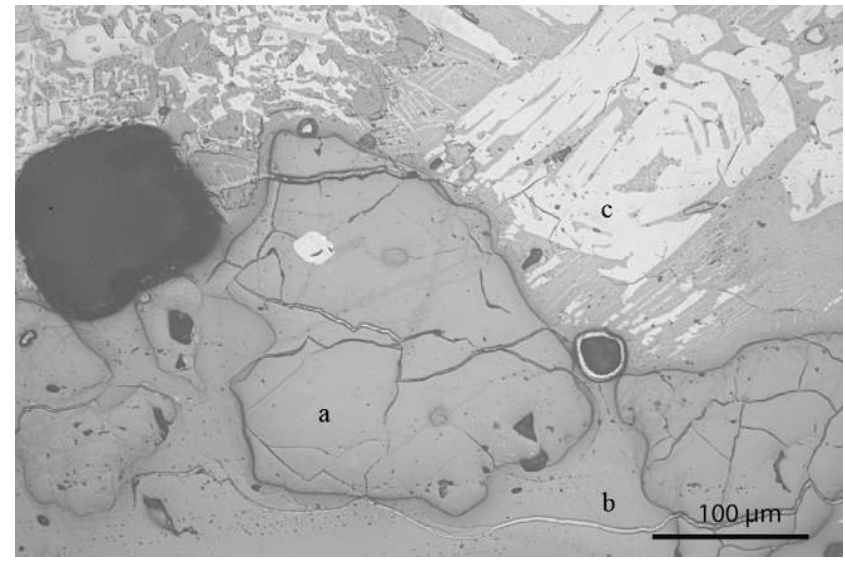

Figure 3 : Matériau argilo-sableux partiellement fondu : restes de grains de quartz (a) dans une matrice vitreuse (b), près d'une zone scoriacée contenant de la fayalite (c).

Figure 3: Siliceous zone partially molten: some relitic quartz (a) in a vitreous matrix (b), near a slaggy zone contained fayalitic crystal (c).

jet (Dunikowski et al., 1996). Lorsque ces croûtes tombent en dehors du foyer, au cours du martelage, elles sont appelées « battitures ». Par contre, lorsqu'elles tombent ou s'écoulent à l'intérieur du foyer, elles vont intégrer la scorie située au fond. Ces croûtes peuvent présenter différents états de transformation, depuis la croûte identique à la battiture et formée essentiellement de magnétite (Fig. 4), jusqu’à un état de fusion totale et de disparition dans la scorie : ne restent alors que des paquets de wüstite plus ou moins compacts selon le degré de dissolution dans la scorie.

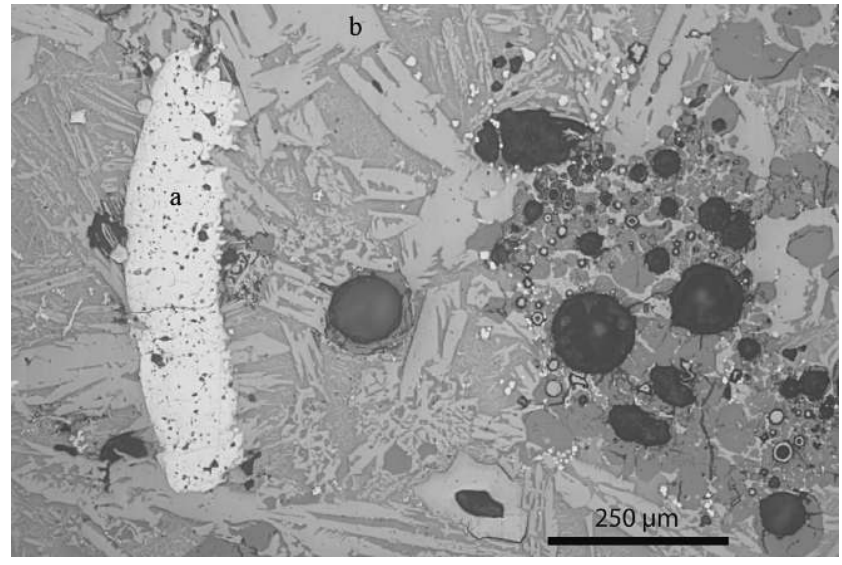

Figure 4 : Exemple d'une croûte d'oxydes de fer (a) en cours de fusion dans une matrice scorifiée de type SGD (cristaux de fayalite (b) dans une matrice vitreuse).

Figure 4: Example of iron oxydes crust (a) partially molten in a SGD type slag matrix (fayalitic crystals (b) in a vitreous matrix). 


\section{Le métal en cours de fusion}

La chute des particules métalliques dans le foyer lors de la chauffe du métal peut être accidentelle, résulter de défauts dans la pièce ou de mauvaises manipulations. Cette origine est soulignée parfois par des bordures rectilignes ou des contours anguleux, ainsi que par des reliques d'écrouissage ou de lignes de soudure. Certaines de ces particules ont brûlé sous l'action du feu (Fig. 5). Elles s'agglomèrent et s'altèrent à l'intérieur du culot sous l'action de la chaleur et des silicates.

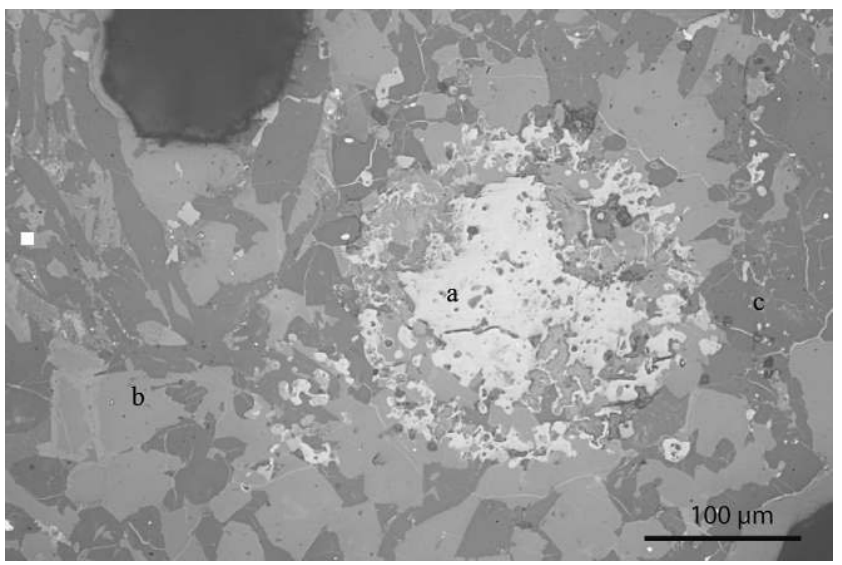

Figure 5 : Exemple de métal partiellement fondu (a) dans de la scorie (cristaux de fayalite [b] et de leucite [c])

Figure 5: Example of iron metal melted (a) in slag (fayalitic [b] and leucitic [c] crystals).

\section{Les zones entièrement scoriacées}

Lorsque toutes les matériaux présentés ci-dessus ont totalement fondus dans le fond du foyer, le culot est composé uniquement d'une matrice scoriacée qui peut être très homogène au niveau de la texture interne. Toutes les phases minéralogiques qu'on observe sont issues de la recristallisation d'un mélange qui était liquide quand le foyer était en fonctionnement. Il s'agit d'oxydes de fer telles que la wüstite ou la magnétite (fusion soit des croûtes, soit du fer métallique, Fig. 6), ou de phases silicatées dont la nature minéralogique sera en relation directe avec la nature des ajouts qui ont été utilisés (essentiellement de la fayalite quand l'ajout est de type sable, Fig. 7).

\section{Les analyses chimiques}

À cause de l'hétérogénéité de composition des culots, l'analyse globale (i.e. représentative de la globalité du déchet) n'est pertinente que dans le cas où ces analyses pourront être comparées à celles d'autres déchets sidérurgiques (scories de réduction ou de forge) provenant, au mieux du même site, voire du même district sidérurgique.

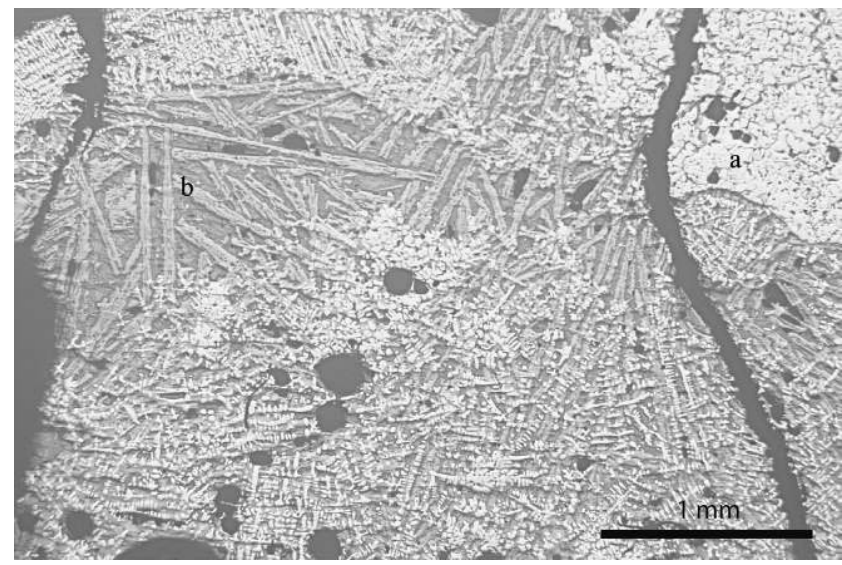

Figure 6 : scorie de type SGD formée principalement par de wüstite (a). Les lattes de fayalite (b) sont visibles entre les accumulations de wüstite.

Figure 6: SGD type slag mainly formed by wüstite (a). Fayalitic crystals (b) are visible between wüstite accumulations.

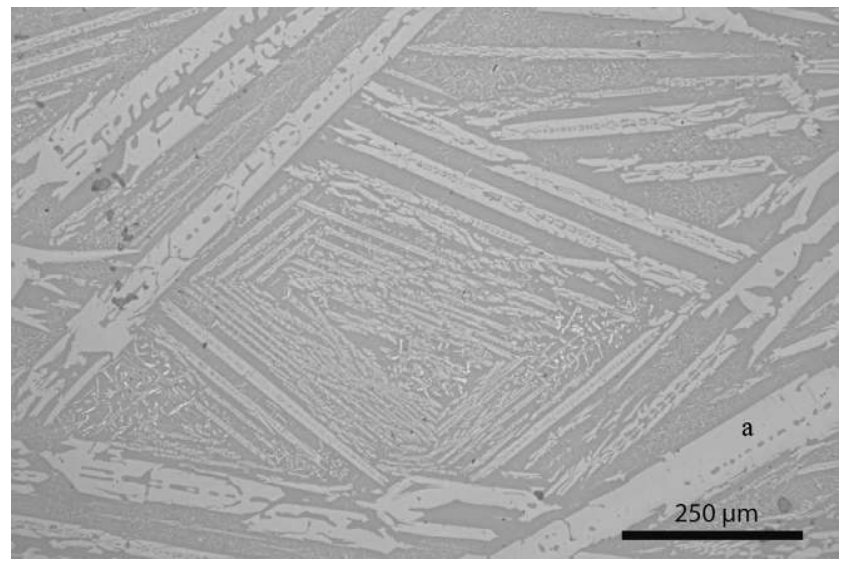

Figure 7 : Scorie de type SGD formée principalement par de la fayalite (a).

Figure 7: SGD type slag mainly formed by fayalitic crystals (a).

Les analyses chimiques zone par zone sont plus délicates à réaliser car il faut prendre soin d'analyser une seule zone à la fois. Il est donc difficile de réaliser des analyses sur poudre (analyses par ICP ou fluorescence X), car il faut scier avec beaucoup de précision le matériau. Néanmoins, ces analyses permettent d'obtenir des données sur la teneur en éléments en traces, ce qui peut être très intéressant pour certaines interprétations. Une autre méthode d'analyse peut être appliquée: des analyses réalisées au moyen de l'EDS (Energy Dispersive System) sur MEB ou au moyen de la fluorescence X par réflexion. Les surfaces analysées seront celles qui auront été observées au microscope optique. Ces analyses vont servir à déterminer la nature des différents matériaux qui vont entrer dans la composition du déchet. 
Néanmoins, avec le MEB, seuls les éléments majeurs (au dessus de $1 \%$ poids) peuvent être analysés.

\section{UNE ÉTUDE DE CAS : LES CULOTS DE FORGE DU SITE DU PUY-DE-GrÂCE}

Le site du Puy-de-Grâce (Perpezac-le-Noir, Corrèze) a été fouillé en 1996 sous la direction de Christine Best (AFAN). Il correspond à une occupation de la Tène finale (seconde moitié $\mathrm{II}^{\mathrm{e}}$ siècle-première moitié $\mathrm{I}^{\mathrm{er}}$ siècle a.C.) caractérisée principalement par une activité métallurgique ${ }^{1}$. Les niveaux d'occupation sont riches en rejets de travaux de forge : $120 \mathrm{~kg}$ de scories en culot (pour la moitié complets), $25 \mathrm{~kg}$ de scories argilo-sableuses, $6,5 \mathrm{~kg}$ de déchets métalliques et $5 \mathrm{~kg}$ de fragments de parois de foyer. Les sols de travail du niveau d'occupation le plus récent ont livré aussi des centaines de grammes de battitures; leur répartition spatiale différentielle permet de localiser une des zones d'activité à proximité de l'un des foyers découverts. Des petits objets, comme par exemple des fibules en fil de fer, sont très probablement fabriqués. Tous ces types de déchets ont été quantifiés et décrits; chacun apporte un certain nombre d'informations sur les techniques de forge et les produits utilisés ou fabriqués. Seule est présentée ici l'étude des culots de forge ${ }^{2}$.

\section{L'importance de l'étude macroscopique : le culot image de la zone de chauffe à l'intérieur du foyer}

Les caractéristiques dimensionnelles rapportées au positionnement de l'axe de la tuyère permettent de définir quatre familles de culot : les culots de plan subcirculaire (50\%); les culots de plan ovale allongés dans l'axe de la tuyère (20 \%); les culots de plan ovale allongés dans l'axe perpendiculaire à la tuyère $(25 \%)$; les culots trapus, également allongés dans l'axe perpendiculaire de celui de la tuyère, mais évoquant une forme de plan rectangulaire avec des angles arrondis (5\%).

La forme générale de la pièce et particulièrement la morphologie du fond renvoie des informations sur la forme, si ce n'est du foyer, au moins de la zone de chauffe. La répétition d'une forme globalement plano-convexe (seuls quelques

1. Les résultats de la fouille archéologique n’ont pas encore été publiés. Le Document Final de Synthèse est déposé au Service régional de l'archéologie du Limousin (Limoges).

2. Le quart des culots de forge a été retrouvé intégré aux niveaux de sol d'occupation correspondant au dernier état de conservation du site (Phase 5, La Tène finale). Tous les autres proviennent du remplissage de structures excavées (fosses et fossés) rattachées aux phases d'occupation précédente (30\% à la phase à la phase 2 et $40 \%$ à la phase 4 , également datées de La Tène finale). culots ont un fond plat) indique que la base du foyer de forge est un aménagement concave. La présence récurrente d'une strate de terre cuite grise adhérente au fond convexe révèle que la base du foyer est soigneusement aménagée. De nombreux culots conservent la trace de l'accrochage contre une paroi, sous la forme d'un fragment du revêtement argileux dont la surface est fortement vitrifiée. Les culots se forment donc à proximité immédiate de la paroi qui porte l'orifice de ventilation et parfois en contact avec elle. Lépaisseur maximale mesurée sur les culots signifie que la tuyère n'est pas positionnée au raz du fond du foyer de forge, mais au moins à $65 \mathrm{~mm}$ au-dessus. La forme en culot, l'absence de trace d'outil, la présence de fragments arrachés de paroi et de revêtement de fond de foyer, sont autant d'éléments qui montrent que la scorie est retirée après refroidissement, c'està-dire après l'arrêt du foyer.

Lexistence d'au moins trois groupes morphologiques pourrait être attribuée à l'utilisation d'autant de foyers différents. Mais il peut aussi bien s'agir de réaménagements du fond d'un même foyer : ainsi, la forme de la base du foyer pourrait être adaptée aux dimensions des pièces travaillées.

Les caractères dimensionnels de chacun des groupes morphologiques évoluent de manière similaire et continue : aucun sous-groupe dimensionnel n'est perceptible (Fig. 8). La morphologie et la dimension des culots dépendent probablement de la quantité de déchets produits, donc de métal travaillé. Les culots du Puy-de-Grâce sont tous de petites et moyennes dimensions (de quelques dizaines à quelques centaines de grammes et entre 50 et $150 \mathrm{~mm}$ de plus grand axe). Nous pouvons seulement remarquer que les culots trapus semblent être uniquement de petite taille. L'absence de culots de grandes dimensions signifieraient que seuls des fers de taille petite ou moyenne sont travaillés, et qu'on ne chauffe pas de gros volume en une seule fois.

\section{Les matériaux constituant le culot : l'enregistrement de types de travail et de cycles de travaux}

\section{Des faciès minéralogiques stratifiés}

L'observation morphologique globale, celle de la section des pièces et l'examen pétrographique au microscope de cette dernière permettent d'identifier la nature des matériaux entrant dans la composition des différentes catégories de culots (matrice scoriacée grise dense, matières argilo-sableuses, fer métallique et oxydes de fer, supra) et de définir les différentes associations minéralogiques que constituent ces matériaux après leur fusion et leur recristallisation. Pour le Puy-de-Grâce, cette étude a porté sur presque la moitié des pièces complètes (40 sur 92). On définit ainsi : 


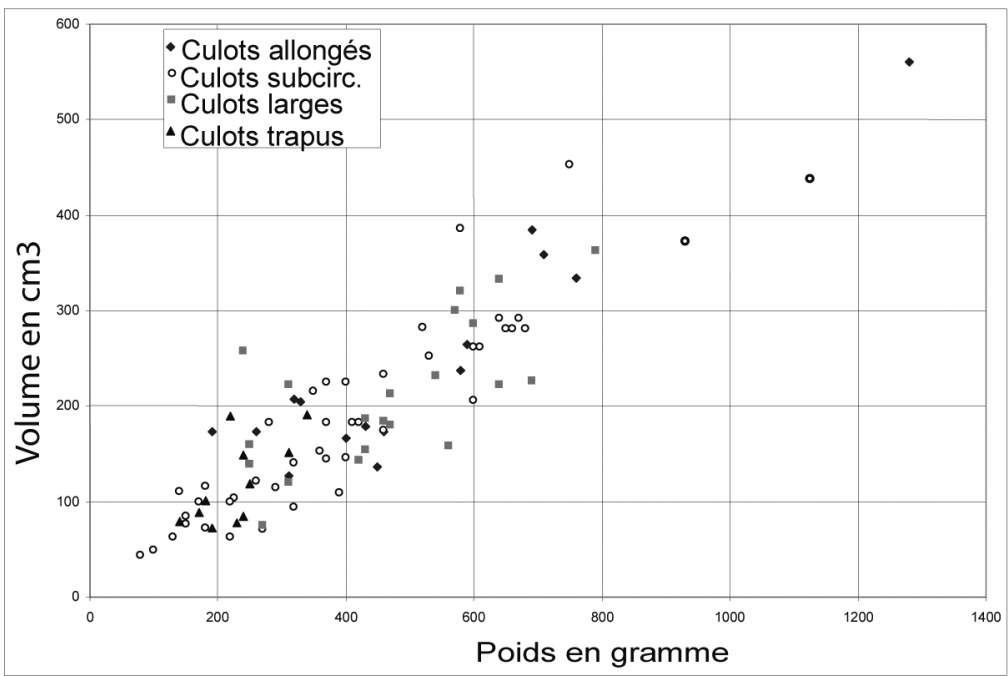

Figure 8 : Représentation des quatre types morphologiques des scories en culot du Puy-de-Grâce dans le diagramme volume / poids

Figure 8: Representation of the four types of Puy-deGrâce slags in the diagram volumelwheight.
- un faciès silicaté, constitué d'une matrice fayalitique, riche en wustite (SGD de Serneels 2004);

- un faciès silicaté fayalitique pauvre ou sans wustite (SGD de Serneels);

- un faciès riche en éléments argilo-sableux plus ou moins fondu, intégrés à une matrice plus ou moins cristallisée (SAS de Serneels);

- un faciès riche en parcelles de fer métallique plus ou moins largement oxydé (SFR de Serneels).

Ces faciès ont été globalement décrits plus haut. Presque tous les culots observés montrent un agencement en strates superposées ou imbriquées de ces faciès. Le nombre de strates varie de 1 à 5 . L'occurrence la plus fréquente (17 sur 40) est celle comptant trois strates. La variété des séquences d'associations de faciès pétrographiques est assez grande (14 agencements différents). On peut toutefois les rassembler en quatre groupes :

- groupe 1 : faciès silicaté riche ou non en wustite surmonté, le plus souvent, par une strate de faciès argilo-sableux (SGD seul / SGD + SAS / 30\% des culots);

- groupe 2: constitué d'une association non stratifiée des faciès du groupe précédent (SGD + SAS / 20\% des culots);

- groupe 3 : faciès silicaté riche ou non en wustite avec strate de faciès riche en fer (SGD + SFR +/- SAS / 22,5\% des culots);

- groupe 4 : faciès riche en fer dominant avec strate ou plages intégrées de faciès silicaté (SFR +/- SGD / 25\% des culots).

\section{Une stratification enregistrant des types de travaux}

Les culots du groupe 4, caractérisés par une perte de fer abondante pourraient être reliés à des travaux pour lesquels le fer est porté à hautes températures : compactage de fer non encore forgeable, agglomération de fers de récupération (dans ces deux cas, il s'agit de préparer la matière première), accidents de chauffe entraînant le détachement de petites parcelles.

Les culots des groupes 1 et 2 correspondraient plutôt aux déchets d'opérations de forgeage utilisant des ajouts pour limiter l'oxydation du fer au cour des cycles de chauffagemartelage (travaux de compactage et de mise en forme) ou pour éliminer la croûte d'oxydes de surface afin par exemple de procéder à des opérations de soudure. Les culots du groupe 3 pourraient résulter d'une association de ces différents types d'opérations.

\section{Accumulation successive ou ségrégation?}

Létude microscopique a également porté sur l'origine de la stratification de ces culots : sont-ils le résultat d'une accumulation de matériaux liée à une succession de phases de travail ou peuvent-ils correspondre à une ségrégation par différence de densité, une grande majorité des culots présentant une strate dense en partie inférieure? La délimitation précise de chaque zone et la détermination de leur nature ont montré que ces culots résultaient pour l'essentiel de l'accumulation progressive de matériaux, même si quelques écoulements de scories ont pu être observées. Du fait sans doute de la différence de viscosité, les coulures s'insèrent entre les strates inférieures mais ne semblent pas se mélanger à elles. De même, les strates inférieures ne montrent pas de traces de refusion au contact des strates sus-jacentes. Il semble donc qu'on puisse affirmer que la stratification des matériaux à l'intérieur des culots résultent plus vraisemblablement d'accumulation d'apports successifs que d'une ségrégation par densité. 


\section{Nature des matériaux argilo-sableux : l'apport de l'étude minéralogique et chimique}

L'étude minéralogique accompagnée d'analyses chimiques permet, lorsque la conservation des restes argilo-sableux est suffisante, de déterminer leur nature. Ainsi, dans les culots du Puy-de-Grâce, de très nombreux fragments de roches peu transformés sont présents en partie supérieure. Par observation comparative, il ne s'agit pas de fragments de parois de foyer qui auraient pu se fragmenter au cours des opérations de forge et tomber dans le foyer.

Deux types de roches naturelles ont été identifiés : d'une part, des fragments contenant de très gros cristaux de quartz et de feldspath potassique qui peuvent être caractéristiques d'une roche nommée " pegmatite » (Fig. 9). D’autre part, un autre type de roche présente l'association minéralogique suivante : des cristaux de quartz et de feldspath potassique, des micas de type biotite, et d'autres minéraux plus rares, appelés " accessoires » tels que des ilménites $\left(\mathrm{TiO}_{2}\right)$ ou des zircons $\left(\mathrm{ZrSiO}_{4}\right)$. Cet assemblage peut correspondre à une roche métamorphique appelée "gneiss ». Ces deux types de roches sont présents dans l'environnement géologique immédiat du site.

Les scories en culot sont cependant constituées majoritairement par de la scorie aux phases minéralogiques néoformées (wüstite, fayalite, leucite essentiellement). Dans ce cas, les matériaux apportés se sont totalement déstructurés et ont fondu, se mélangeant ainsi en une masse quasi-homogène.

L'identification des matériaux initiaux doit alors être effectuée selon une autre méthode d'investigation. Celle-ci est basée sur la connaissance de la composition chimique des zones scoriacées. Plusieurs analyses chimiques sont réalisées sur chaque type de zone des culots. L'analyse des mêmes reliques d'ajouts présents sur les battitures peut également aider à l'interprétation (Dunikowski et al., 1996).

Il s'agit de réaliser un traitement statistique des données en créant des diagrammes : les éléments chimiques constitutifs des analyses sont comparés les uns aux autres de manière à observer l'évolution respective de leur teneur. Si deux éléments chimiques proviennent du même matériau, leurs teneurs respectives devraient évoluer de la même manière. Dans un diagramme binaire, ces deux éléments chimiques présenteront une corrélation positive. Des éléments chimiques apportés par deux matériaux initiaux totalement différents ne montreront pas la même évolution : dans le diagramme, il n'y aura pas de corrélation entre ces deux éléments, ou la corrélation sera négative (effet de mélange). Pour qu'un matériau soit identifiable, il faut que son apport soit majoritaire. Le fait que certains matériaux ajoutés aient pu être identifiés grâce aux fragments peu transformés nous aide beaucoup dans l'interprétation des diagrammes.

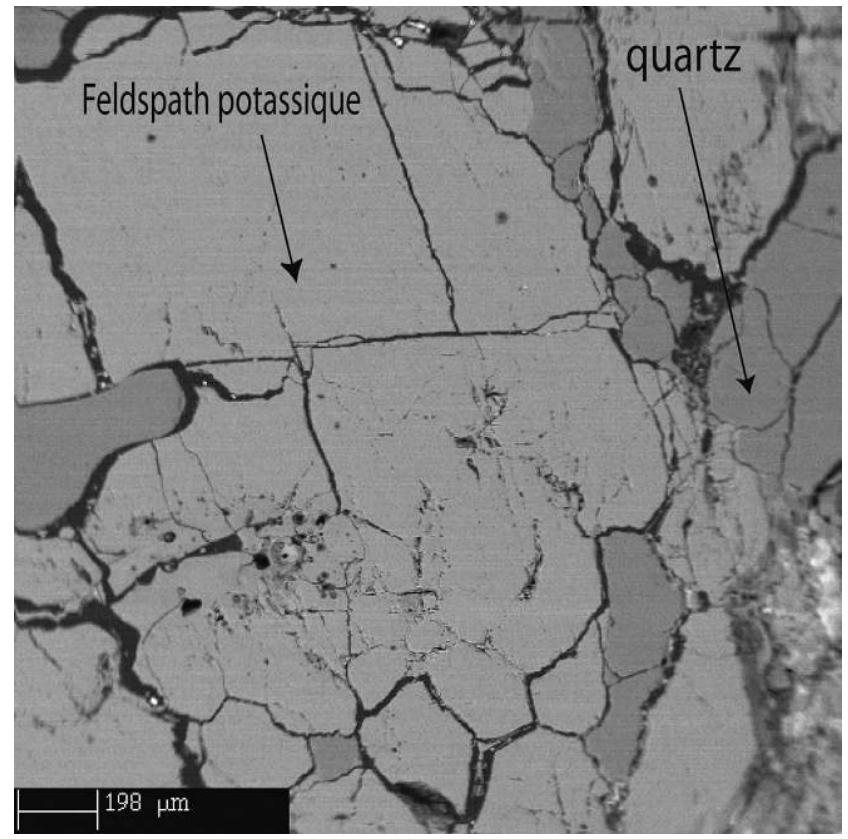

Figure 9: Observation au microscope électronique d'un fragment de pegmatite non fondu dans une scorie argilo-sableuse du Puyde-Grâce.

Figure 9: Electronic microscope observation of a piece of non molten pegmatite in a siliceous slag of the Puy-de-Grâce.

Dans le diagramme $\mathrm{Al}_{2} \mathrm{O}_{3} / \mathrm{TiO}_{2}$, les points sont très fortement corrélés positivement (Fig. 10). Ceci reflète généralement la présence d'une phase argileuse. Les argiles contiennent aussi de la silice. Ceci se traduit également par une corrélation positive entre ces deux éléments, visibles dans les scories avec métal et dans les battitures: le ou les ajouts utilisés sont donc essentiellement de nature argileuse. Se peut-il que cette argile soit de même nature que celle utilisée à la confection des parois du foyer? Le rapport $\mathrm{Al}_{2} \mathrm{O}_{3} /$ $\mathrm{SiO}_{2}$ similaire dans les parois et dans les déchets analysés semble apporter des éléments de réponse dans ce sens. Il est possible alors que cette argile provienne de l'environnement géologique immédiat du site.

Dans la partie argilo-sableuse des culots, on note un enrichissement en $\mathrm{SiO}_{2}$ par rapport à $\mathrm{Al}_{2} \mathrm{O}_{3}$ ce qui traduit un apport supplémentaire en silice par un autre matériau. Il s'agit des fragments de gneiss et de pegmatite. L'apport principal de la silice dans la partie supérieure des culots vient par conséquent des fragments centimétriques présents dans le foyer, et notamment ceux d'origine pegmatitique qui contiennent de gros fragments de quartz; l'apport de $\mathrm{SiO}_{2}$ est donc plus important dans ce cas-là que l'apport de $\mathrm{Al}_{2} \mathrm{O}_{3}$. Par ailleurs, face à l'apport minéral lié à la présence des fragments de roche et dans une moindre mesure à celle 


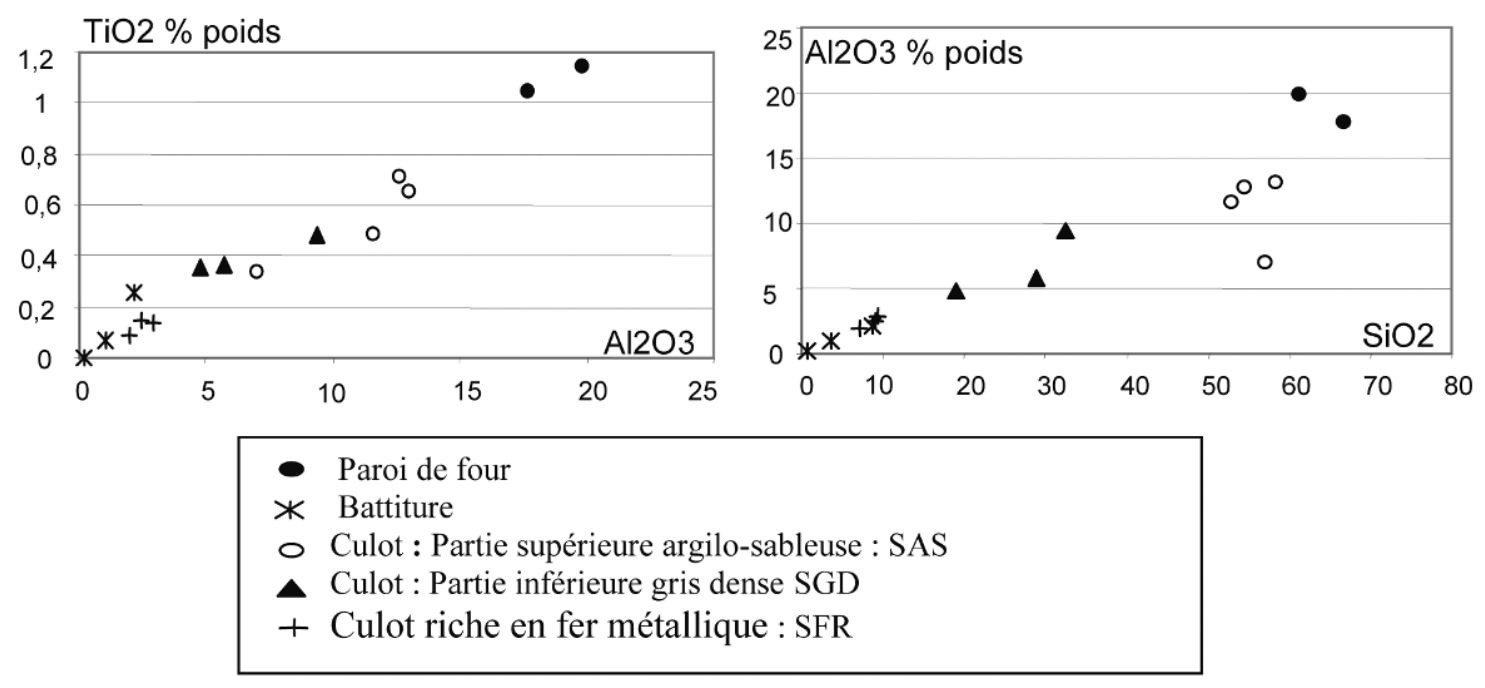

Figure 10 : Diagrammes de corrélation entre éléments chimiques permettant des hypothèses quant à la nature de l'ajout dans le culot. Â gauche, corrélation positive entre $\mathrm{TiO}_{2}$ et $\mathrm{Al}_{2} \mathrm{O}_{3}$ indiquant la présence d'argile. À droite, la plus forte en $\mathrm{SiO}_{2}$ dans la partie supérieure des culots indique une présence plus importante dans cette zone de grains de quartz. Matériaux analysés provenant du site du Puy-de-Grâce.

Figure 10: Correlation diagrams between chemical elements lead to propose some hypothesis concerning the nature of addition present in slag. To the left, the positive correlation between TiO2 and Al2O3 indicates the clay presence. To the right, the most content of $\mathrm{SiO}_{2}$ in the upper part of the slag indicates a most important contribution of quartz. Analysed materials come from Puy-de-Grâce site.

de l'argile, l'apport lié aux cendres de charbon de bois n'est pas visible chimiquement.

\section{L'évaluation de la quantité de fer perdu}

La démarche a été introduite par V. Serneels (2004). Il s'agit dans une première étape de calculer la masse de fer perdu dans l'ensemble des déchets métallurgiques. L'étude de la composition minéralogique des culots et de l'agencement stratigraphique des différents faciès permet d'évaluer la part de fer métallique potentielle piégée dans les culots. La part quantitative de chacune des strates de matériaux (argilo-sableuse SAS, scories silicaté grise dense SGD, scorie ferreuse riche en fer SFR) dans l'ensemble du corpus de culots étudiés peut être évaluée à partir des sections observées (Tableau 1). La composition minéralogique et chimique de chacune de ces catégories de matériaux permettant de connaitre leur teneur moyenne en fer (entre 10 et $20 \%$ du poids pour SAS; entre 35 et $50 \%$ du poids pour SGD; entre 55 et $70 \%$ du poids pour SFR), il est ensuite possible d'arriver à une estimation de la proportion du poids de fer contenu dans l'ensemble des culots : ici entre 31,5 et $44,5 \%$ du poids total des culots. Les $98 \mathrm{~kg}$ de culots recueillis sur le site renferment donc entre 30 et $44 \mathrm{~kg}$ d'équivalent fer (sous forme métallique ou dans les oxydes de fer). Si l'on ajoute les
$20 \mathrm{~kg}$ de scories denses informes (7 à $10 \mathrm{~kg}$ d'équivalent fer) et les $25 \mathrm{~kg}$ de scories argilo-sableuses (3 à $5 \mathrm{~kg}$ d'équivalent fer), ainsi que les $6,5 \mathrm{~kg}$ de déchets métalliques, on obtient une masse de fer perdue à l'intérieur de la masse de déchets récoltés lors de la fouille, de l'ordre de 45 à $65 \mathrm{~kg}$.

Toutefois tous les déchets produits au cours du fonctionnement des différentes phases d'ateliers n'ont probablement pas été retrouvés (limites du site non atteintes, sols d'occupation non conservés en intégralité). Et il est impossible d'estimer quelle est la part de ce qui a été retrouvée lors de la fouille par rapport à la masse de déchets réellement produits.

Quoi qu'il en soit, et parce que les conditions de conservation des sites et des artefacts sur les sites ne permettront jamais d'avancer des chiffres précis, c'est l'ordre de grandeur qu'il est important de mettre en avant. La quantité de fer perdu lors des travaux de forge ne représente probablement qu'une petite proportion de la quantité de fer réellement travaillée3. Aussi, si une cinquantaine ou une soixantaine de kilogrammes de fer a été perdu et piégée dans la masse de déchets retrouvés, c'est qu'au moins plus d'une centaine

3. Même si les proportions de perte de fer au cours du travail de forge sont encore loin de pouvoir être évaluée (la question est complexe au regard de la diversité des travaux pratiqués et des savoir-faire techniques mis en œuvre). 


\begin{tabular}{|l|l|l|l|}
\hline $\begin{array}{l}\text { Catégories de faciès pétrographi- } \\
\text { ques }\end{array}$ & $\begin{array}{l}\text { Part du faciès dans le corpus de } \\
\text { culots }\end{array}$ & $\begin{array}{l}\text { Part d'équivalent fer par type de } \\
\text { faciès }\end{array}$ & $\begin{array}{l}\text { Part d'équivalent fer dans le } \\
\text { corpus de culots }\end{array}$ \\
\hline SAS scorie argilo-sableuse & $23 \%$ & 5 à $20 \%$ & 1 à $5 \%$ \\
\hline SGD scorie grise dense & $67 \%$ & 35 à $50 \%$ & 23 à $33 \%$ \\
\hline SFR scorie ferreuse & $9 \%$ & 55 à $75 \%$ & 5 à $7 \%$ \\
\hline \multicolumn{2}{|l|}{ Moyenne de la part de fer dans le corpus de culos } & & 29 à $45 \%$ \\
\hline \multicolumn{2}{|l|}{ Poids de fer } & $98 \mathrm{~kg}$ & 28,5 à $44 \mathrm{~kg}$ \\
\hline
\end{tabular}

Tableau 1 : Intervalle de teneurs en fer pour les différents types de matériaux.

Table 1: Range of iron content in the different types of material.

de kilogrammes de fer a été travaillée. Compte tenu des éléments dont nous disposons, il n'est pas possible d'être plus précis. Mais une masse de 100 ou $200 \mathrm{~kg}$ de fer, c'est déjà l'équivalent d'une production de 1000 objets de $100 \mathrm{~g}$ (comme des couteaux), de 5000 objets de $20 \mathrm{~g}$ (comme de fibules de fer par exemple).

\section{DisCUSSION ET CONCLUSION}

L'apport de l'étude des scories en culot à la compréhension et à la restitution des activités de post-réduction est essentiel. Leur observation morphologique détaillée, l'enregistrement des informations métrologiques, l'examen de leur structure interne et l'identification des matériaux qui les composent, l'application d'investigations analytiques, fournissent des informations variées qui sont d'autant plus faciles à collecter que la taille des pièces se prête à ce type d'investigations.

$1^{\circ}$ ) L'aménagement du foyer de forge, son mode de conduite et les gestes techniques du forgeron, sont autant de paramètres qui interagissent et influent sur l'aspect morphologique extérieur et sur la nature du déchet qui se forme dans le foyer de forge. On peut donc penser qu'un même procédé de travail conditionne les mêmes types d'aménagements du foyer de forge, les mêmes modes de conduite et les mêmes gestes techniques du forgeron, et par conséquent aboutit à des déchets comparables. Dans cette hypothèse, l'étude des différentes catégories de culots de forge pourrait nous renseigner sur la nature des travaux réalisés et sur leur processus de formation.

$2^{\circ}$ ) Formes et dimensions du culot peuvent nous donner une image de la forme et des dimensions de la zone de chauffe. D'après les manuels de forgerons, les dimensions du feu doivent toujours être adaptées aux dimensions de la pièce à chauffer.

$3^{\circ}$ ) Une scorie en culot se forme au cours d'une série d'opérations qui débute avec l'allumage du foyer et se finit avec l'arrêt du feu. Chaque pièce correspond donc à une sorte de cycle ou d'unité de travail à la fin duquel le culot est systématiquement enlevé du foyer. Ce cycle de travail peut correspondre à une unité de temps, tout autant qu'à une unité de travail (ensemble de gestes techniques dans une suite opératoire cohérente). Ainsi, les strates observées à l'intérieur du culot peuvent représenter les traces de ces cycles successifs de chauffage. D'une certaine manière, le culot représente une accumulation de matière tombée dans le fond du foyer au cours d'un cycle de travail entre la mise en route de la forge et son arrêt.

$\left.4^{\circ}\right)$ Les culots seraient les témoins des travaux de forge nécessitant des hautes températures $\left(950^{\circ} \mathrm{C}\right.$ à $\left.1450^{\circ} \mathrm{C}\right)$. Ces températures de chauffage génèrent nécessairement une oxydation à chaud plus active et des risques plus élevés de brûler le métal, ce qui nécessite implicitement l'utilisation d'un adjuvant de protection des surfaces. De plus, les travaux qui demandent des chauffages successifs, fréquents et prolongés, ne feront qu'accroître le phénomène d'oxydation. La présence de parcelles de fer susceptibles de provenir de la fusion partielle d'une pièce de métal ne peut qu'abonder dans le sens de chaudes à hautes températures. A contrario, les travaux qui requièrent des températures de martelage plus basses devraient entraîner une oxydation moins importante et donc ne nécessiteraient pas le recours à des adjuvants. Il n'est donc pas incohérent de penser que les travaux de forge à plus basses températures ne généreraient pas de culot.

$5^{\circ}$ ) Sur le site du Puy-de-Grâce, les culots contenant principalement des parcelles de métal sont minoritaires (culots du groupe 4). Les travaux susceptibles de générer de telles pertes sont potentiellement nombreux et il ne s'agit pas nécessairement d'opérations de compactage de fer brut, hypothèse qui a souvent été avancée dans des études récentes ${ }^{4}$. On pourrait donc aussi considérer qu'ils ne représentent que des accidents de chauffe ayant entraîné des pertes

4. Au demeurant, l'interprétation doit aussi se fonder sur l'analyse minéralogique et chimique de la matrice silicatée et sur la structure du métal, bien que celle-ci ait été le plus généralement fortement modifiée par les températures atteintes. 
en fer. Lors des chauffes sans accident, les culots ne devraient contenir que des pertes en oxydes de fer. Les culots des groupes 1 et 2 seraient donc produits lors d'opérations pendant lesquels des ajouts sont déposés ou projetés sur la pièce de fer, soit pour limiter la formation d'une croûte d'oxydes, soit pour l'éliminer (en vue de soudures par exemple). Ces opérations consistent vraisemblablement en des séries de chauffes suivies de martelage, pour compacter la pièce et la mettre en forme. À chaque remise au feu de la pièce de fer préalablement enduite d'ajouts sableux ou argileux, des gouttes du liquide silicaté formé sur la surface vont s'écouler au fond du foyer. Mais au cours de ces opérations, la perte de fer métallique est rare, voire insignifiante. De nombreux types de travaux de forge engendrent de tels cycles de chauffage/martelage.

$\left.6^{\circ}\right)$ On remarque aussi que les surépaisseurs argilo-sableuses des culots ne contiennent que peu de métal, ou d'oxyde de fer. Dans la mesure où on considère qu'ils proviennent de l'utilisation d'adjuvants destinés à protéger le fer de l'oxydation lors de la chauffe à hautes températures, leur présence indique que l'ajout est directement jeté sur la pièce alors qu'elle se trouve dans le foyer de forge. Ils illustreraient alors à un excès d'ajout. La quasi-absence d'oxydes de fer dans ces surépaisseurs argilo-sableuses indiquerait que le but est atteint ou que les oxydes formés sur le métal sont très peu nombreux car la chauffe est rapide (petit objet par exemple), voire qu'ils ne tombent pas dans le foyer. D'une manière générale, ce geste semble souvent être le dernier avant l'arrêt du foyer.

$7^{\circ}$ ) La quantification générale des matériaux constituants le culot corrélée aux informations chimiques permet de fournir des ordres de grandeur sur la quantité de fer métallique perdu (sous forme d'oxydes ou sous forme brûlée), donc indirectement sur un ordre de grandeur de la quantité de fer travaillé.

On signalera enfin que les autres informations collectées par l'étude tout aussi détaillée des autres catégories de déchets (scories argilo-sableuses, petites coulures de scories, différentes catégories de battitures et de déchets métalliques) doivent être corrélées avec celles fournies par l'étude des scories en culot pour permettre l'interprétation de l'activité de l'atelier. Aucune interprétation paléométallurgique ne peut s'appuyer sur l'étude d'une seule catégorie de matériel, ni sur une seule méthode d'investigation. C'est la convergence de l'ensemble des résultats apportés par les études archéologiques comme archéométriques, qui permet d'asseoir et d'orienter les interprétations aux différentes échelles d'étude (produits, gestes techniques, procédés, organisation des ateliers et de la production...). C'est cet assemblage de déchets qui caractérise l'atelier et permet de le comparer à d'autres.
Cependant, l'état d'avancement des recherches ne permet pas encore de déterminer avec certitude la nature des travaux réalisés dans un atelier (épuration, agglomération, soudure, martelage), ni d'assigner avec précision un type de déchets à un type de travail. Ces questions trouveront réponse à la fois dans la poursuite des recherches, ainsi que par le développement de reconstitutions expérimentales dont les déchets seront analysés avec autant de précision que ceux que l'on retrouve sur les sites archéologiques.

\section{Bibliographie}

Dunikowski, C., Leroy M., Merluzzo P. et Ploquin A., 1998. L'atelier de forge de Nailly (Yonne), contribution à l'étude des déchets métallurgiques, Revue Archéologique de l'Est et du Centre-Est, 47, p. 97-121.

Eschenlohr, L., Friedil, V., Robert-Charrue Linder, C., Senn, M. et Develier-Courtételle, 2007. Un habitat rural mérovingien. Métallurgie du fer et mobilier métallique, Cahier d'archéologie jurassienne, 14, Porrentruy, 355 p.

LEROY, M., 1997. La sidérurgie en Lorraine avant le haut fourneau. L'utilisation du minerai de fer oolithique en réduction directe, Paris, CNRS, coll. " Monographies du CRA », 306 p.

Mangin, M. (dir.) 2004. Le Fer, Paris, Errance, coll. « Archéologiques », $239 \mathrm{p}$.

Mauvilly, M. et al., 1998. Sévaz "Tudinges » : chronique d'un atelier de métallurgistes du début de La Tène dans la Broye, Archéologie Suisse, 21, n 4, p. 144-154.

Mc Donnell, J. G., 1987. The study of Early Iron Smithing residues, in Scott, B. G. and Cleere, H. (dir.), The Crafts of the Blacksmith, Belfast, Symposium CSA, 1984, p. 47-52.

Orengo, L., 2003. Forges et forgerons dans les habitats laténiens de la Grande Limagne d'Auvergne. Fabrication et consommation de produits manufacturés en fer en gaule à l'Age du Fer, Monographies Instrumentum, 26, Montagnac, ed. Monique Mergoil, 325 p.

Orengo, L., Frenee, E. et Fluzin, P., 2000. Un atelier de forge de l'Âge du Fer au "Bois du Jarrier 3" commune de LaCelle-sur-Loire (Nièvre), Archéologie et archéométrie, in : Feugères, M., et Gustin, M. (dir.), Iron, Blacksmiths and Tools, Ancient European Crafts, Acts of the Instrumentum Conference at Podsreda (Slovenia), 1999, ed. Monique Mergoil, 12, Montagnac, p. 45-66.

Ploquin, A., Leroy, M., Merluzzo, P. et Dunikowski, C., 1998. Des déchets métallurgiques, quels indices pour une forge?, in Nicolini, G. et Dieudonné-Glad, N. (éd.), Les métaux antiques: travail et conservation, Actes du colloque de Poitiers 1995, Montagnac, ed. Monique Mergoil, Monographie Instrumentum, 6, p. 145-152. 
Serneels, V., 1993. Archéométrie des scories de fer, Recherches sur la sidérurgie ancienne en Suisse occidentale, Cahiers d'Archéologie Romande, 61, Lausanne, 1993, 240 p.

Serneels, V., 1999. Le travail du fer et les objets en fer, in Chardron-Picault, P. et Pernot, M. (dir.), Un quartier antique d'artisanat métallurgique à Autun (Saône et Loire), le site du lycée militaire, Paris, Éditions de la Maison des Sciences de l'Homme, p. 201-218.

Serneels, V., 2004. Les scories et les matériaux associés, in Anderson T. et al., Des Artisans à la campagne, Carrières de meules, forge et voie gallo-romaine à Châbles, Archéologie fribourgeoise 19, Academic Press, Fribourg, p. 104-146. 